\title{
MicroCT-based evaluation of the trabecular bone quality of different implant anchorage sites for masticatory rehabilitation of the maxilla
}

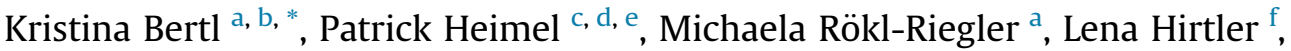 \\ Christian Ulm ${ }^{a}$, Werner Zechner ${ }^{\mathrm{a}}$ \\ a Division of Oral Surgery, University Clinic of Dentistry, Medical University of Vienna (Head: Prof. Christian Ulm, DMD, MD), Sensengasse 2a, \\ A-1090 Vienna, Austria \\ ${ }^{\mathrm{b}}$ Department of Periodontology, Faculty of Odontology, University of Malmö, Sweden \\ c Karl Donath Laboratory for Hard Tissue and Biomaterial Research, Division of Oral Surgery, Medical University of Vienna, Austria \\ d Austrian Cluster for Tissue Regeneration, Vienna, Austria \\ e Ludwig Boltzmann Institute for Clinical and Experimental Traumatology, Vienna, Austria \\ ${ }^{\mathrm{f}}$ Center for Anatomy and Cell Biology, Department of Systematic Anatomy, Medical University of Vienna, Austria
}

\section{A R T I C L E I N F O}

\section{Article history:}

Paper received 27 October 2014

Accepted 8 April 2015

Available online $\mathrm{xxx}$

Keywords:

Dental implants

Maxilla

Micro-computed tomography

Zygoma

\begin{abstract}
A B S T R A C T
In the severely atrophied maxilla, implant anchorage in the zygomatic bone is considered a viable alternative to conventional dental implants with preceding bone augmentation procedures. The present microCT-based study compared the trabecular bone quality of the maxilla and zygomatic bone. MicroCT scanning was conducted in 12 halves of cadaver heads ( 5 male, 7 female) with edentulous, atrophied maxillae. Relevant trabecular bone quality parameters were determined in the anterior and posterior maxilla and in the zygomatic bone and compared by region and sex. Any difference in mean values between the anterior maxilla and the zygomatic bone was insignificant. Comparison of both with the posterior maxilla presented significantly higher values for bone volume fraction, surface density, and trabecular thickness and number, and significantly lower values for specific bone surface, structure model index, and trabecular separation. A significant sex-specific difference was not detected. The present microCT-based analysis is, to the best of our knowledge, the first intra-individual comparison of different implant anchorage sites for masticatory rehabilitation of the maxilla. The trabecular compartment of the zygomatic bone offered bone quality and, thus, an implant bed comparable with those of the anterior maxilla, and both were superior to the posterior maxilla.
\end{abstract}

(C) 2015 European Association for Cranio-Maxillo-Facial Surgery. Published by Elsevier Ltd. All rights

reserved.

\section{Introduction}

Since their introduction by Brånemark et al. (1969), dental implants for tooth replacement have enjoyed great success. Modifications of design, material, and surface properties have resulted in an implant survival rate of approximately $93 \% 10$ years after prosthetic rehabilitation (Pjetursson et al., 2004). However, dental implants have limitations in patients with severe alveolar bone resorption after tooth loss. In particular, in the maxilla, where pneumatization of the maxillary sinus significantly adds to

\footnotetext{
* Corresponding author. Division of Oral Surgery, University Clinic of Dentistry, Medical University of Vienna, Sensengasse 2a, A-1090 Vienna, Austria. Tel.: +43 1 40070 4101; fax: +431400704709.

E-mail address: kristina.bertl@meduniwien.ac.at (K. Bertl).
}

the total bone loss, more complex treatments are necessary to provide sufficient implant anchorage; bone augmentation procedures (eg, sinus floor elevation) are well established and predictable (Del Fabbro et al., 2008; Corbella et al., 2013). However, they are associated with higher patient risk and morbidity (i.e., in extensive atrophic areas probably even with extra-oral bone retrieval donor site morbidity), higher costs, and prolonged treatment times; this creates opportunities for alternatives such as zygomatic implants.

Zygomatic implants were initially introduced for masticatory rehabilitation of the maxilla in cancer or trauma patients with severe defects in the upper jaw (eg, after hemi-maxillectomy) (Weischer et al., 1997). In these cases, the zygomatic bone offered an alternative site for implant anchorage. Between two and four zygomatic implants, together with or without standard dental implants, in the anterior maxilla can be used for immediate or 
delayed prosthetic loading protocols (Malevez et al., 2004; Balshi et al., 2009; Candel-Marti et al., 2012; Davo et al., 2013; Esposito and Worthington, 2013; Goiato et al., 2014). Although only approximately one-third of zygomatic implants are anchored within bone (Nkenke et al., 2003; Corvello et al., 2011; Balshi et al., 2012), they present high survival rates. A recent systematic review (Goiato et al., 2014) evaluated 1541 zygomatic implants and reported a survival rate of $97.86 \%$ after 36 months; first 10 -year results presented a cumulative survival rate of $95.12 \%$ (Aparicio et al., 2014). Hence, zygomatic implants are a predictable alternative to restorations with standard dental implants with preceding bone augmentation procedures. However, for detailed evaluation of the advantages and disadvantages of these treatment options, highquality randomized controlled clinical trials are still warranted (Esposito and Worthington, 2013).

Since the introduction of zygomatic implants, three surgical techniques have been developed. In the original one, according to Brånemark (1998), the implant is positioned inside the maxillary sinus. This was followed by the slot technique (Stella and Warner 2000) with a smaller access, and an exteriorized (or extrasinus) approach (Migliorança et al., 2006), where the implant is positioned outside the maxillary sinus. The exteriorized technique has advantages in terms of reduced invasiveness, fewer sinus complications, less surgical time, fewer surgical steps, and most often an increased osteotomy length in the zygomatic bone (Corvello et al., 2011; Aparicio et al., 2013; Chrcanovic et al., 2013). The technique chosen depends on the surgeon's preferences and, most of all, the patient's anatomy: an anterior maxillary wall which is only slightly concave would be best suited by the original technique, while a pronounced concavity suggests the exteriorized technique (Aparicio et al., 2010, 2013; Aparicio, 2011; Chrcanovic et al., 2013).

The increase in our knowledge of maxillary and mandibular bone quantity and quality facilitated - and still facilitates - relevant improvements in standard dental implants, resulting in improved osseointegration and survival rates. For zygomatic bone, studies on its quantity are available (Uchida et al., 2001; Nkenke et al., 2003; Van Steenberghe et al., 2003; Corvello et al., 2011; Balshi et al., 2012), but data on its quality are still scarce and, except for one histological study by Nkenke et al. (2003), are restricted to Japanese populations (Kato et al., 2004, 2005; Yoshino et al., 2007). Possible differences due to ethnicity (Uchida et al., 2001; Nkenke et al., 2003; Liu et al., 2011; Kim et al., 2013b; Putman et al., 2013) and the technique applied for bone analysis (histomorphometry vs. micro-computed tomography (microCT)) (Chappard et al., 2005; Bonnet et al., 2009), and the lack of an intraindividual comparison with the maxilla, triggered the present microCT-based study to assess the trabecular bone quality of the zygomatic bone and compare it with the anterior and posterior maxilla.

\section{Material and methods}

\subsection{Specimen fixation and preparation}

The study protocol was approved by the ethics committee of the Medical University of Vienna (EK-Nr. 1713/2013). Twelve halves of human phenol-formaldehyde-fixed cadaver heads ( 5 male, $7 \mathrm{fe}-$ male) with edentulous, atrophied maxillae were included. The deceased had bequeathed their bodies to the Anatomical Institute of the Medical University of Vienna for medical-scientific research and training purposes. The fixation of the whole human corpses was performed by perfusion of the vascular system with a phenolformaldehyde solution ( $4.1 \%$ phenol and $2.1 \%$ formalin in water) for at least $24 \mathrm{~h}$. Detailed data on age, time elapsed since tooth loss, regular wear of mandibular dentures, and intake of any medication affecting bone metabolism were not available. Before microCT scanning, the mandible was removed, and the upper parts were reduced as far as possible to the maxilla and the zygomatic bone, with care taken to preserve the naso-palatine canal for midpoint determination.

\subsection{MicroCT scanning and definition of the volume of interest}

All samples were scanned for structural analysis by microCT (Viscom XT9190-THP) (transmission tube, digital detector, voxel size $35 \mu \mathrm{m}^{3}, 120 \mathrm{kV}, 400 \mu \mathrm{A}$; filter, $0.75 \mathrm{~mm}$ copper; exposure time, $1400 \mathrm{~ms}$; zoom factor, 3300).

The scans were oriented so that the skull was upright and the hard palate was horizontal, with Amira 5.4.2 software (FEI Visualization Sciences Group, Burlington, MA, USA). Three volumes of interest (VOI) were selected: (1) anterior and (2) posterior maxilla, and (3) zygomatic bone. The limits of the maxillary VOI were set as follows: (1) canalis incisivus; (2) distal border of the tuberosity; (3) floor of the nasal cavity in the anterior and palatal roof in the posterior region; and (4) at half of the height between the most caudal point of the alveolar process and the palatal roof, the width of the alveolar process was used as the medial limit (minimum, $7 \mathrm{~mm}$ ). At the distal aspect of the canine, considering mean values of tooth widths, the maxilla was separated into an anterior and posterior part. The limits of the zygomatic bone were set as follows: (1) sutura zygomaticomaxillaris; (2) most caudal point of the orbita; and (3) distal border of the processus frontalis ossis zygomatici.

\subsection{Assessment of trabecular bone quality parameters}

The three VOIs were analysed by Definiens Developer XD 2.1 (Definiens AG, Munich, Germany). Trabecular and cortical bone were differentiated as follows. First, the void volume outside the bone was separated from the void volume inside the bone. For this separation, 'region growing' was performed with the bone as a seed. The bone was coated with an 8-pixel thick $(560 \mu \mathrm{m})$ layer of a temporary class. Regions of void, which were contained inside this temporary class, were classified as void inside the bone, and the remaining void volume outside this class was classified as void outside the bone. 'Surface-tension-constrained region growing' was performed with void outside the bone as a seed into the temporary class. The surface tension prevented the void outside the bone from growing into gaps in the bone. The temporary class, which remained after this growing step, was classified as void inside the bone. The sinus volume was automatically added to the void outside the bone, based on the relation between its large volume and relatively small contact length toward bone.

A Canny edge detection (Canny, 1986) was performed to help with the separation of cortical and trabecular bone. 'Surface-tension-constrained region growing' was performed using the void outside of the bone as a seed. Starting from this seed, the cortical region was allowed to grow into bone with a high bone volume fraction in the surrounding volume (i.e., cortical bone but not trabecular bone). The growing was additionally constrained by the previously detected edges. In the therefrom-resulting trabecular bone area, the following trabecular bone quality parameters were assessed:

- Total volume (TV; $\left.\mathrm{mm}^{3}\right)$

- Bone volume (BV; $\mathrm{mm}^{3}$ )

- Bone surface (BS; $\mathrm{mm}^{2}$ )

- Bone volume fraction (BV/TV; \%) 
- Bone surface density (BS/TV; $1 / \mathrm{mm})$

- Specific bone surface (BS/BV; $1 / \mathrm{mm})$

- Connectivity density (Conn.D; $1 / \mathrm{mm}^{3}$ )

- Structure model index (SMI (Hildebrand and Ruegsegger, 1997);

0 for parallel plates and 3 for cylindrical rods)

- Trabecular thickness (Tb.Th; $\mathrm{mm}$ )

- Trabecular separation (Tb.Sp; mm)

- Trabecular number (Tb.N; $1 / \mathrm{mm})$

The segmentation results were exported as binary images of the trabecular VOI within the bone. These binary images were used to define the region of interest (ROI) in Image $1.48 \mathrm{v}$ software (Schneider et al., 2012). The following measurements were performed with BoneJ 1.3.11 software (Doube et al., 2010). The ROI was defined with 'analyze particles', and the results were loaded into the ROI manager. This ROI was used for measuring Tb.Th and Tb.Sp. BS was measured by means of the 'isosurface'tool without down-sampling. Before Conn.D was measured, 'purify' was used for reduction, excluding small, unconnected parts of the trabecular structure, resulting in a single connected object in the image, which is required for obtaining correct results with the connectivity measurement. The SMI was also measured without down-sampling; otherwise, the default settings were used.

\subsection{Statistical analysis}

Normal distribution of the data was proven graphically ( $Q-Q$ plot). For comparison between the regions (anterior and posterior maxilla, zygomatic bone), one-way ANOVA was used, and, in cases of significance $(p<0.05)$, the LSD post-hoc test was applied. Sex comparison was performed by independent $t$-test, and correlations were analysed by Pearson's correlation coefficient. Statistical analysis was performed with a statistical program (SPSS Version 19.0 , Chicago, IL, USA), and $p$-values $<0.05$ were considered as statistically significant.

\section{Results}

\subsection{Trabecular bone quality-comparison between regions}

Trabecular bone quality parameters of all regions are summarized in Table 1. Throughout the results, the anterior maxilla and the zygomatic bone presented comparable values and, for all parameters, except for Conn.D, were significantly different from the posterior maxilla. For the parameters BV/TV, BS/TV, Tb.Th, and $\mathrm{Tb} . \mathrm{N}$, the posterior maxilla presented significantly lower values, while for the parameters BS/BV, SMI, and Tb.Sp, the values were significantly higher. In particular, for BV/TV, huge differences were detected between the assessed regions, with the maximum value of the posterior maxilla being similar to the minimum values of the anterior maxilla and the zygomatic bone. The SMI presented, for all regions, a more rod-like trabecular structure, especially for the posterior maxilla, with a value close to the maximum of three.

\subsection{Trabecular bone quality-comparison between the sexes}

The sex comparison of the trabecular bone quality parameters is presented in Table 2. The parameters BV/TV, BS/TV, Tb.Th, and Tb.N presented, in all regions, higher mean values for males but the differences were not statistically significant. Further, the mean values of BS/BV were lower for males, with a significant difference for the zygomatic bone. SMI and Tb.Sp presented rather

Table 1

Trabecular bone quality parameters of the anterior and posterior maxilla and of the zygomatic bone.

\begin{tabular}{|c|c|c|c|c|c|c|}
\hline \multirow[t]{3}{*}{ Parameter } & & \multicolumn{5}{|l|}{ Region } \\
\hline & & \multicolumn{2}{|l|}{ Maxilla } & \multirow[t]{2}{*}{ Zygoma } & \multirow[t]{2}{*}{$p$-value (one-way ANOVA) } & \multirow[t]{2}{*}{$p$-value (post-hoc LSD test) } \\
\hline & & Anterior & Posterior & & & \\
\hline \multirow[t]{2}{*}{ BV/TV (\%) } & Mean $\pm S D$ & $27.15 \pm 7.9$ & $13.54 \pm 3.4$ & $26.79 \pm 7.4$ & $<0.001$ & $\begin{array}{l}<\mathbf{0 . 0 0 1}^{\mathrm{a}} \\
<\mathbf{0 . 0 0 1}^{\mathrm{b}}\end{array}$ \\
\hline & Min; max & $17.12 ; 45.46$ & $9.15 ; 18.32$ & $17.34 ; 42.86$ & & $0.894^{c}$ \\
\hline \multirow[t]{2}{*}{$\mathrm{BS} / \mathrm{TV}(1 / \mathrm{mm})$} & Mean \pm SD & $4.49 \pm 1.1$ & $2.81 \pm 0.8$ & $3.83 \pm 0.7$ & $<0.001$ & $\begin{array}{r}<\mathbf{0 . 0 0 1}^{\mathrm{a}} \\
\mathbf{0 . 0 0 8}^{\mathrm{b}}\end{array}$ \\
\hline & Min; $\max$ & $3.22 ; 6.38$ & $1.75 ; 4.39$ & $2.94 ; 4.90$ & & $0.075^{\mathrm{c}}$ \\
\hline \multirow[t]{2}{*}{$\mathrm{BS} / \mathrm{BV}(1 / \mathrm{mm})$} & Mean \pm SD & $17.05 \pm 3.8$ & $20.96 \pm 3.6$ & $14.71 \pm 2.2$ & $<0.001$ & $\begin{array}{r}\mathbf{0 . 0 0 6}^{\mathrm{a}} \\
<\mathbf{0 . 0 0 1}^{\mathrm{b}}\end{array}$ \\
\hline & Min; max & $10.76 ; 23.91$ & $15.24 ; 27.40$ & $10.85 ; 17.18$ & & $0.090^{c}$ \\
\hline \multirow[t]{2}{*}{ Conn.D $\left(1 / \mathrm{mm}^{3}\right)$} & Mean \pm SD & $6.31 \pm 3.0$ & $4.46 \pm 2.4$ & $5.76 \pm 2.0$ & 0.196 & - \\
\hline & Min; $\max$ & $3.66 ; 13.90$ & $1.54 ; 8.73$ & $3.16 ; 9.58$ & & \\
\hline \multirow[t]{2}{*}{ SMI } & Mean \pm SD & $2.17 \pm 0.3$ & $2.78 \pm 0.3$ & $2.23 \pm 0.4$ & $<0.001$ & $\begin{array}{l}<\mathbf{0 . 0 0 1}^{\mathrm{a}} \\
<\mathbf{0 . 0 0 1}^{\mathrm{b}}\end{array}$ \\
\hline & Min; max & $1.79 ; 2.90$ & $2.11 ; 3.24$ & $1.46 ; 2.68$ & & $0.638^{c}$ \\
\hline \multirow[t]{2}{*}{$\operatorname{Tb} . \operatorname{Th}(\mathrm{mm})$} & Mean $\pm S D$ & $0.256 \pm 0.04$ & $0.217 \pm 0.03$ & $0.261 \pm 0.04$ & 0.014 & $\begin{array}{l}\mathbf{0 . 0 1 6}^{\mathrm{a}} \\
\mathbf{0 . 0 0 8}^{\mathrm{b}}\end{array}$ \\
\hline & Min; max & $0.217 ; 0.378$ & $0.195 ; 0.270$ & $0.224 ; 0.347$ & & $0.775^{\mathrm{c}}$ \\
\hline \multirow[t]{2}{*}{$\mathrm{Tb} . \mathrm{Sp}(\mathrm{mm})$} & Mean \pm SD & $0.590 \pm 0.13$ & $0.893 \pm 0.14$ & $0.626 \pm 0.13$ & $<0.001$ & $\begin{array}{l}<\mathbf{0 . 0 0 1}^{\mathrm{a}} \\
<\mathbf{0 . 0 0 1}^{\mathrm{b}}\end{array}$ \\
\hline & Min; $\max$ & $0.369 ; 0.763$ & $0.684 ; 1.153$ & $0.457 ; 0.840$ & & $0.506^{\mathrm{c}}$ \\
\hline \multirow[t]{2}{*}{ Tb.N(1/mm) } & Mean \pm SD & $1.051 \pm 0.20$ & $0.624 \pm 0.14$ & $1.024 \pm 0.20$ & $<0.001$ & $\begin{array}{l}<\mathbf{0 . 0 0 1}^{\mathrm{a}} \\
<\mathbf{0 . 0 0 1}^{\mathrm{b}}\end{array}$ \\
\hline & Min; $\max$ & $0.730 ; 1.392$ & $0.451 ; 0.926$ & $0.750 ; 1.370$ & & $0.707^{c}$ \\
\hline
\end{tabular}

Significant $p$-values are presented in bold.

BS/BV: specific bone surface; BS/TV: bone surface density; BV/TV: bone volume fraction; Conn.D: connectivity density; SMI: structure model index; Tb.N: trabecular number; Tb.Sp: trabecular separation; Tb.Th: trabecular thickness.

a Comparison between anterior and posterior maxilla.

b Comparison between posterior maxilla and zygomatic bone.

c Comparison between anterior maxilla and zygomatic bone. 
Table 2

Trabecular bone quality parameters of the anterior and posterior maxilla and of the zygomatic bone analysed on sex differences (independent $t$-test).

\begin{tabular}{|c|c|c|c|c|}
\hline & & Female $(n=7)$ & Male $(n=5)$ & $p$-value \\
\hline \multirow[t]{3}{*}{ BV/TV (\%) } & Anterior maxilla & $24.27 \pm 6.4$ & $31.17 \pm 8.7$ & 0.143 \\
\hline & Posterior maxilla & $12.27 \pm 2.3$ & $15.31 \pm 4.0$ & 0.127 \\
\hline & Zygoma & $24.52 \pm 6.8$ & $29.96 \pm 7.6$ & 0.222 \\
\hline \multirow[t]{3}{*}{$\mathrm{BS} / \mathrm{TV}(1 / \mathrm{mm})$} & Anterior maxilla & $4.15 \pm 1.2$ & $4.97 \pm 0.9$ & 0.229 \\
\hline & Posterior maxilla & $2.60 \pm 0.7$ & $3.10 \pm 0.9$ & 0.292 \\
\hline & Zygoma & $3.79 \pm 0.8$ & $3.88 \pm 0.6$ & 0.841 \\
\hline \multirow[t]{3}{*}{$\mathrm{BS} / \mathrm{BV}(1 / \mathrm{mm})$} & Anterior maxilla & $17.31 \pm 3.6$ & $16.68 \pm 4.5$ & 0.792 \\
\hline & Posterior maxilla & $21.21 \pm 3.6$ & $20.61 \pm 4.0$ & 0.791 \\
\hline & Zygoma & $15.75 \pm 1.3$ & $13.26 \pm 2.3$ & 0.041 \\
\hline \multirow[t]{3}{*}{ Conn.D $\left(1 / \mathrm{mm}^{3}\right)$} & Anterior maxilla & $5.98 \pm 3.8$ & $6.77 \pm 1.7$ & 0.675 \\
\hline & Posterior maxilla & $3.60 \pm 1.7$ & $5.67 \pm 2.9$ & 0.149 \\
\hline & Zygoma & $6.30 \pm 2.4$ & $4.99 \pm 1.2$ & 0.242 \\
\hline \multirow[t]{3}{*}{ SMI } & Anterior maxilla & $2.21 \pm 0.2$ & $2.11 \pm 0.5$ & 0.594 \\
\hline & Posterior maxilla & $2.84 \pm 0.3$ & $2.69 \pm 0.4$ & 0.467 \\
\hline & Zygoma & $2.22 \pm 0.4$ & $2.25 \pm 0.4$ & 0.911 \\
\hline \multirow[t]{3}{*}{ Tb.Th (mm) } & Anterior maxilla & $0.244 \pm 0.01$ & $0.274 \pm 0.06$ & 0.348 \\
\hline & Posterior maxilla & $0.205 \pm 0.01$ & $0.234 \pm 0.03$ & 0.118 \\
\hline & Zygoma & $0.239 \pm 0.02$ & $0.290 \pm 0.05$ & 0.090 \\
\hline \multirow[t]{3}{*}{ Tb.Sp (mm) } & Anterior maxilla & $0.631 \pm 0.13$ & $0.532 \pm 0.11$ & 0.206 \\
\hline & Posterior maxilla & $0.911 \pm 0.12$ & $0.866 \pm 0.18$ & 0.602 \\
\hline & Zygoma & $0.624 \pm 0.14$ & $0.629 \pm 0.13$ & 0.954 \\
\hline \multirow[t]{3}{*}{ Tb.N (1/mm) } & Anterior maxilla & $0.993 \pm 0.24$ & $1.133 \pm 0.11$ & 0.254 \\
\hline & Posterior maxilla & $0.598 \pm 0.10$ & $0.659 \pm 0.18$ & 0.474 \\
\hline & Zygoma & $1.015 \pm 0.22$ & $1.035 \pm 0.18$ & 0.867 \\
\hline
\end{tabular}

Data are presented as mean $\pm S D$, and significant $p$-values are presented in bold.

BS/BV: specific bone surface; BS/TV: bone surface density; BV/TV: bone volume fraction; Conn.D: connectivity density; SMI: structure model index; Tb.N: trabecular number; Tb.Sp: trabecular separation; Tb.Th: trabecular thickness.

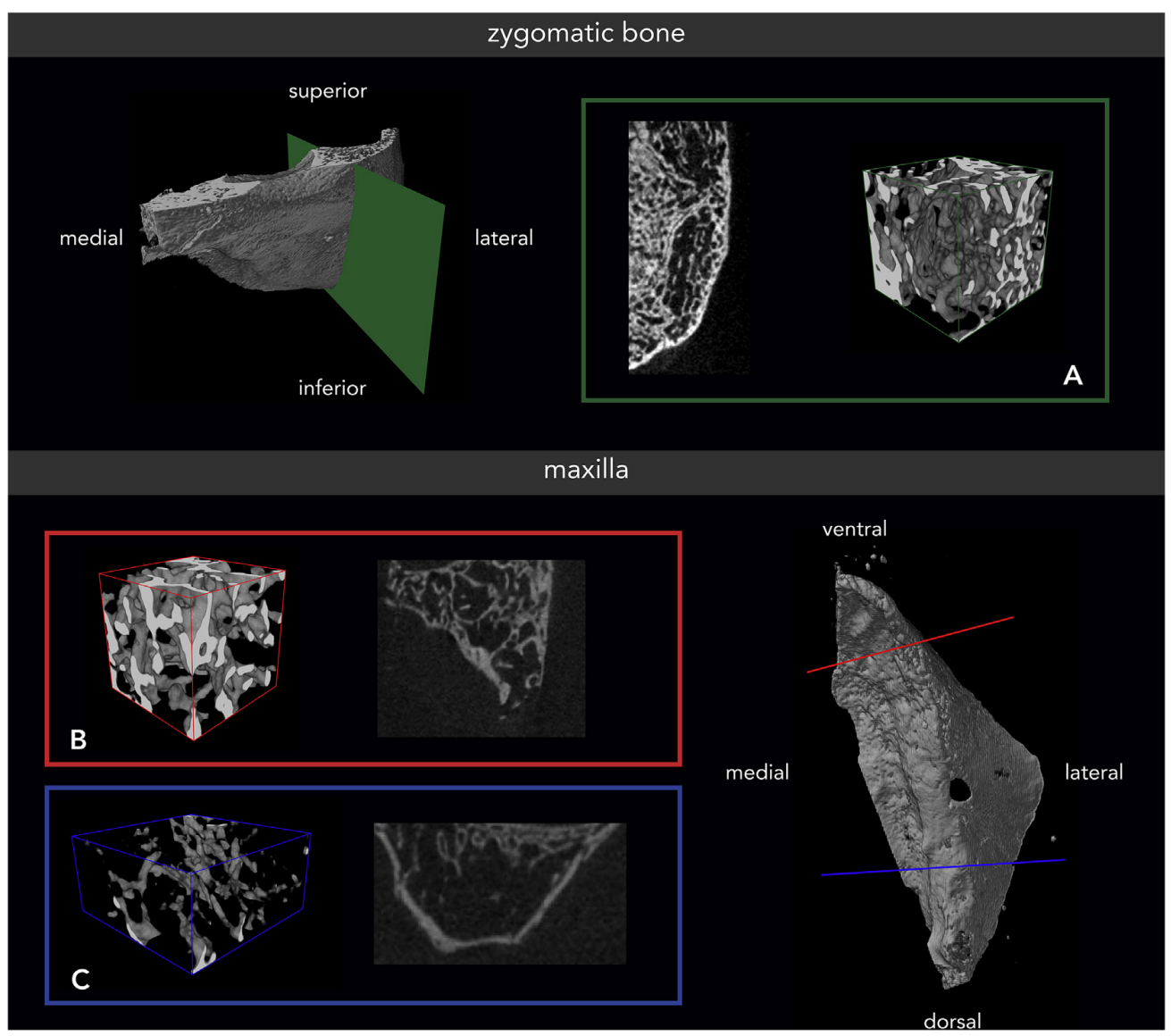

Fig. 1. Zygomatic and maxillary bone of a female specimen with a lower range bone volume fraction (BV/TV). (A) Zygomatic bone (green) with a BV/TV of $25.82 \%$; (B) anterior maxilla (red) with a BV/TV of $18.78 \%$; and (C) posterior maxilla (blue) with a BV/TV of $9.94 \%$. The 3D reconstructions are presenting trabecular bone only. 
comparable values, especially for the zygomatic bone. Conn.D presented, for males, insignificantly higher values in the maxilla and insignificantly lower values in the zygomatic bone. Fig. 1 shows zygomatic and maxillary bone specimens from a female with a lower range $\mathrm{BV} / \mathrm{TV}$, and Fig. 2 shows bone specimens from a male with a higher range $\mathrm{BV} / \mathrm{TV}$.

\subsection{Correlations between regions and between parameters}

$\mathrm{BV} / \mathrm{TV}$ correlated between the posterior maxilla and the zygomatic bone (rho $=0.632, p=0.028$ ), and BS/TV and BS/BV correlated within the maxilla, that is between the anterior and posterior regions $(\mathrm{BS} / \mathrm{TV}$, rho $=0.760, p=0.004$; $\mathrm{BS} / \mathrm{BV}$, rho $=0.710, p=0.010$ ). All other parameters presented insignificant correlations between regions $(p>0.058$; data not shown).

Correlations between the parameters divided by region are presented in Appendix 1. In all regions, BV/TV correlated positively with Tb.Th and Tb.N and negatively with Tb.Sp. These correlations presented statistical significance, except for Tb.Th in the posterior region. Further, BV/TV correlated significantly positively with BS/ TV in the posterior maxilla and in the zygomatic bone. Conn.D correlated in all regions significantly positively with BS/TV and Tb.N, as well as negatively with Tb.Sp, but missed significance in the anterior maxilla. Finally, Tb.Sp correlated in all regions significantly negatively with Tb.N.

\section{Discussion}

For masticatory rehabilitation of the maxilla, various implant anchorage sites are possible. Knowledge of their bone structure supports clinical decision-making and developments in implant technology. The cortical and trabecular structure of the maxilla has been well investigated. An attractive alternative, which is increasingly chosen in patients with a severely atrophied maxilla to avoid extensive patient morbidity and cost-intensive bone augmentation procedures, is the zygomatic bone. However, data on its bone quality, especially in direct comparison with the maxilla, are still scarce. Hence, the present microCT-based study aimed to provide a comparison of the quality of the trabecular compartment, which is essential for implant osseointegration, between the maxilla and the zygomatic bone. Trabecular bone parameters of the anterior and posterior maxilla and of the zygomatic bone were evaluated in 12 human cadaver heads. While the trabecular bone quality was comparable between the anterior maxilla and the zygomatic bone, the bone of the posterior maxilla was significantly inferior in quality.

The available lengths of zygomatic implants vary from 30 to $52.5 \mathrm{~mm}$, but only about one-third of the total implant length is regularly osseointegrated into the zygomatic bone (Nkenke et al., 2003; Corvello et al., 2011; Balshi et al., 2012). Still, a recent finite element analysis suggested that at least $15 \mathrm{~mm}$ of zygomatic bone support is required to significantly reduce the resulting stresses on the fixture/bone interface (Romeed et al., 2014). The available

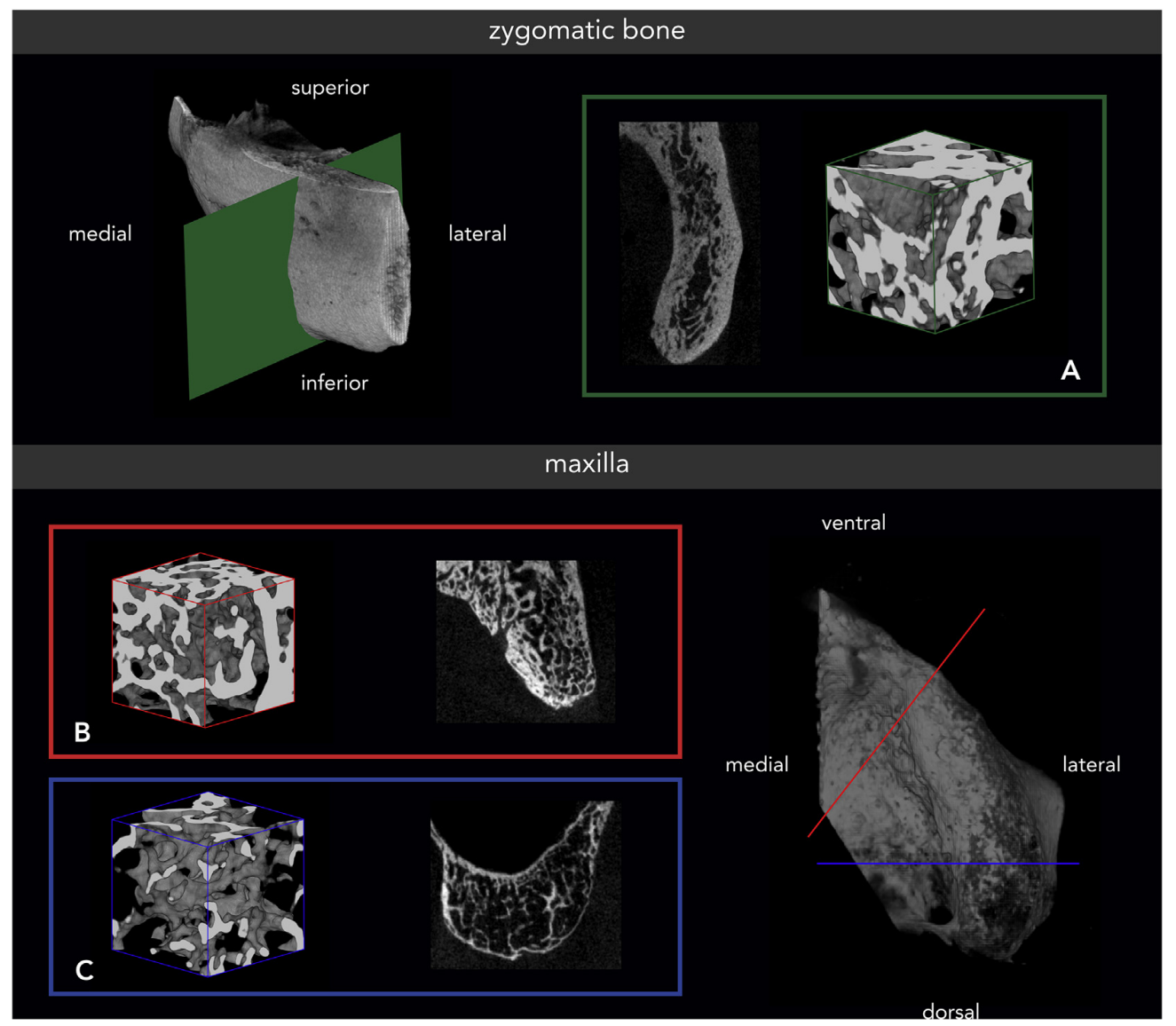

Fig. 2. Zygomatic and maxillary bone of a male specimen with a higher range bone volume fraction (BV/TV). (A) Zygomatic bone (green) with a BV/TV of 27.03\%; (B) anterior maxilla (red) with a BV/TV of 45.46\%; and (C) posterior maxilla (blue) with a BV/TV of $17.96 \%$. The 3D reconstructions are presenting trabecular bone only. 
quantity of zygomatic bone at the implant anchorage site is dependent on various factors such as the placement of one or two implants/site, the insertion technique, and the patient's ethnicity, sex, and body height. Mean bone-to-implant contact in zygomatic bone was evaluated at between 14.0 and $16.5 \mathrm{~mm}$ (Nkenke et al., 2003; Van Steenberghe et al., 2003; Balshi et al., 2012). The original Brånemark technique presented a significantly shorter boneto-implant contact $(8.4 \mathrm{~mm})$ compared with the exteriorized technique $(14.1 \mathrm{~mm})$ (Corvello et al., 2011). Further, next to the sex effect with preferences for males, a shorter body height $(<160 \mathrm{~cm})$ was associated with mean values below $10 \mathrm{~mm}$ and a minimum of only $6.4 \mathrm{~mm}$ compared with mean values of $18.8 \mathrm{~mm}$ at a body height above $160 \mathrm{~cm}$ (Uchida et al., 2001).

Next to the amount of available bone, its quality is a major determining factor for primary implant stability, which is mainly achieved by cortical anchorage, and subsequent successful osseointegration, which is primarily supported by the trabecular compartment. Except for one histological analysis (Nkenke et al., 2003), the zygomatic bone quality was assessed, thus far, by three microCT-based trials assessing Japanese specimens (Kato et al., 2004, 2005; Yoshino et al., 2007). A tooth-loss-dependent change in the trabecular structure of the zygomatic bone was described; dentate specimens presented a higher trabecular bone density, thicker trabeculae, and a more plate-like and constantly oriented trabecular structure (Kato et al., 2004; Yoshino et al., 2007). These differences were enforced in the area of the Jugale point (defined as the most concave point between the lateral margin of the upper zygomatic bone and the upper margin of the zygomatic arch), where masseter muscle and fascia temporalis adhere. Hence, the trabecular structure of the zygomatic bone appears to be significantly influenced by occlusal stress and biting forces. In a subsequent study, Kato et al. (2005) confirmed, for edentulous specimens, a higher trabecular bone quality (higher bone volume fraction with thicker and more plate-like structured trabeculae) in the Jugale region, which also represents the apical anchorage site of zygomatic implants. Possible differences due to ethnicity (Nkenke et al., 2003; Liu et al., 2011; Kim et al., 2013b; Putman et al., 2013) and/or body height (Uchida et al., 2001) suggested the present microCT-based analysis in a Caucasian population. Compared with previous studies on edentulous specimens (micro-CT (Kato et al., 2005) and histomorphometric (Nkenke et al., 2003) analyses), a slightly higher bone volume fraction associated with a higher trabecular thickness was detected for the zygomatic bone.

Nkenke et al. (2003) evaluated the trabecular bone quality of the zygomatic bone as poor when compared with the maxilla (Ulm et al., 1999) due to reduced trabecular connectivity. They assumed primary implant stability to be achieved mainly via multi-cortex stabilization (up to 4 times compared with 1 or 2 times for standard dental implants) (Nkenke et al., 2003). To overcome limitations by comparison of separate studies with non-identical study populations (i.e., differences in time since last tooth loss, age, ethnicity, or sex), the present study performed an intra-individual comparison of the trabecular bone quality of the maxillary and zygomatic bone. The posterior maxilla presented reduced trabecular bone quality compared with both the anterior maxilla and the zygomatic bone. All significant differences reflected a poorer trabecular structure in the posterior maxilla - either higher or lower values (see Table 1). Further, the SMI indicated in all regions a mainly rod-like trabecular structure with values above two and closest to three for the posterior maxilla. Between the anterior maxilla and the zygomatic bone, no significant differences were detected for any trabecular bone quality parameter.

Comparison of the results on maxillary trabecular bone quality with previous histological data (Ulm et al., 1999) confirmed a decreasing quality from anterior to posterior regions, but the values of the present study were in general slightly higher (i.e., minor differences for bone volume fraction and more distinct differences for trabecular thickness, separation, and number). Only the region of the first molar assessed by Ulm et al. (1999) presented a higher bone volume fraction compared with our 'posterior' results, which is presumably due to the inclusion of the maxillary tuberosity in the VOI in this study. Both, histology and microCT, represent standard techniques for bone quality evaluation. However, the data are not interchangeably comparable, and microct analysis tends to overestimate the bone volume fraction and trabecular structure (Chappard et al., 2005; Bonnet et al., 2009).

Other available microcT trials on maxillary trabecular bone quality (Fanuscu and Chang, 2004; Kim et al., 2013a) are in overall agreement. Kim et al. (2013a) set a similar VOI by dividing the anterior and posterior regions of the maxilla at the distal aspect of the canine. Minor differences are reasonably explained by a small sample size $(n=4)$ and missing sex information in the study by Kim et al. (2013a). Yet, a clear difference was present for SMI; Kim et al. (2013a) presented a distinctly more plate-like structure, with values between 1 and 1.2, compared with values above 2 in the present study. As Kim's study was performed in Korea, ethnicity might be considered a possible influencing factor.

Comparison with histological (Ulm et al., 1997, 2009; Bertl et al., 2014) and microCT-based (Moon et al., 2004) studies on the mandibular trabecular bone quality presented overall higher values for the mandible, especially in posterior regions.

Any effect of sex on trabecular bone quality - as often shown in other studies (Ulm et al., 1999, 2009; Nkenke et al., 2003; Bertl et al., 2014) - was not detected in any of the assessed regions. In general, a slightly higher quality among males was detected, but differences in mean values were insignificant. Possible confounding factors might be the missing data on age, time elapsed since tooth loss, regular wear of mandibular dentures, and intake of any medication affecting bone metabolism. These missing data represent, at the same time, the main limitation of the present study, although the impact on the results can be considered acceptable, as the primary comparison was intra-individual.

\section{Conclusion}

The first microcT-based data of the zygomatic bone in comparison with the anterior and posterior maxilla in a Caucasian population are presented. While the zygomatic trabecular bone quality was previously suggested to be poor compared with the maxilla (Nkenke et al., 2003), the present intra-individual analysis proved a comparable trabecular bone quality of the zygomatic bone with the anterior maxilla. Further, both were significantly superior to the posterior maxilla, which might contribute to the inferior outcomes of implant survival rate reported for the posterior maxilla (Ferrigno et al., 2002; Weng et al., 2003). Altogether, the description of an equivalent trabecular bone quality of the zygomatic bone to that of the anterior maxilla presents valuable information for further research on zygomatic implants.

\section{Financial support}

No sources of support.

\section{Conflict of interest statement}

No conflict of interests to declare.

\section{Acknowledgements}

The authors thank Mag. Dockner for his support during microCT scanning. All authors have read the manuscript, agreed to its submission to this journal. 


\section{Appendix}

Appendix 1

Correlations between trabecular bone quality parameters divided by region (Pearson's correlation coefficient).

\begin{tabular}{|c|c|c|c|c|c|c|c|c|}
\hline & & $\mathrm{BV} / \mathrm{TV}$ & $\mathrm{BS} / \mathrm{TV}$ & $\mathrm{BS} / \mathrm{BV}$ & Conn.D & SMI & Tb.Th & Tb.Sp \\
\hline \multicolumn{9}{|c|}{ Anterior maxilla } \\
\hline BS/TV & Anterior maxilla & 0.516 & & & & & & \\
\hline $\mathrm{BS} / \mathrm{BV}$ & & -0.507 & 0.457 & & & & & \\
\hline Conn.D & & 0.419 & 0.748 & 0.272 & & & & \\
\hline SMI & & -0.429 & -0.276 & 0.150 & -0.163 & & & \\
\hline Tb.Th & & 0.811 & 0.349 & -0.383 & 0.099 & -0.523 & & \\
\hline Tb.Sp & & -0.756 & -0.508 & 0.351 & -0.657 & -0.082 & -0.292 & \\
\hline Tb.N & & 0.792 & 0.518 & -0.395 & 0.605 & -0.164 & 0.287 & -0.938 \\
\hline \multicolumn{9}{|c|}{ Posterior maxilla } \\
\hline BS/TV & Posterior maxilla & 0.777 & & & & & & \\
\hline BS/BV & & -0.255 & 0.390 & & & & & \\
\hline Conn.D & & 0.543 & 0.578 & 0.077 & & & & \\
\hline SMI & & -0.577 & -0.731 & -0.239 & -0.93 & & & \\
\hline Tb.Th & & 0.563 & 0.301 & -0.331 & 0.162 & -0.405 & & \\
\hline Tb.Sp & & -0.763 & -0.458 & 0.447 & -0.705 & 0.082 & -0.253 & \\
\hline Tb.N & & 0.863 & 0.733 & -0.140 & 0.600 & -0.416 & 0.074 & -0.801 \\
\hline \multicolumn{9}{|l|}{ Zygoma } \\
\hline BS/TV & Zygoma & 0.825 & & & & & & \\
\hline BS/BV & & -0.782 & -0.324 & & & & & \\
\hline Conn.D & & 0.548 & 0.860 & -0.048 & & & & \\
\hline SMI & & -0.702 & -0.640 & 0.508 & -0.442 & & & \\
\hline Tb.Th & & 0.677 & 0.174 & -0.962 & -0.071 & -0.296 & & \\
\hline Tb.Sp & & -0.699 & -0.928 & 0.170 & -0.844 & 0.501 & -0.070 & \\
\hline Tb.N & & 0.827 & 0.991 & -0.339 & 0.828 & -0.726 & 0.163 & -0.899 \\
\hline
\end{tabular}

Significant correlations are presented in bold $(p<0.05)$

BS/BV: specific bone surface; BS/TV: bone surface density; BV/TV: bone volume fraction; Conn.D: connectivity density; SMI: structure model index; Tb.N: trabecular number; Tb.Sp: trabecular separation; Tb.Th: trabecular thickness.

\section{References}

Aparicio C: A proposed classification for zygomatic implant patient based on the zygoma anatomy guided approach (ZAGA): a cross-sectional survey. Eur J Oral Implantol 4: 269-275, 2011

Aparicio C, Manresa C, Francisco K, Aparicio A, Nunes J, Claros P, et al: Zygomatic implants placed using the zygomatic anatomy-guided approach versus the classical technique: a proposed system to report rhinosinusitis diagnosis. Clin Implant Dent Relat Res, 2013

Aparicio C, Manresa C, Francisco K, Ouazzani W, Claros P, Potau JM, et al: The longterm use of zygomatic implants: a 10-year clinical and radiographic report. Clin Implant Dent Relat Res 16: 447-459, 2014

Aparicio C, Ouazzani W, Aparicio A, Fortes V, Muela R, Pascual A, et al: Extrasinus zygomatic implants: three year experience from a new surgical approach for patients with pronounced buccal concavities in the edentulous maxilla. Clin Implant Dent Relat Res 12: 55-61, 2010

Balshi SF, Wolfinger GJ, Balshi TJ: A retrospective analysis of 110 zygomatic implants in a single-stage immediate loading protocol. Int J Oral Maxillofac Implant. 24: $335-341,2009$

Balshi TJ, Wolfinger GJ, Shuscavage NJ, Balshi SF: Zygomatic bone-to-implant contact in 77 patients with partially or completely edentulous maxillas. J Oral Maxillofac Surg 70: 2065-2069, 2012

Bertl K, Subotic M, Heimel P, Schwarze UY, Tangl S, Ulm C: Morphometric characteristics of cortical and trabecular bone in atrophic edentulous mandibles. Clin Oral Implant. Res, 2014

Bonnet N, Laroche N, Vico L, Dolleans E, Courteix D, Benhamou CL: Assessment of trabecular bone microarchitecture by two different $\mathrm{x}$-ray microcomputed tomographs: a comparative study of the rat distal tibia using Skyscan and Scanco devices. Med Phys 36: 1286-1297, 2009

Brånemark PI: Surgery and fixture installation: zygomaticus fixture clinical procedures. Nobel Biocare AB, 1998

Brånemark PI, Adell R, Breine U, Hansson BO, Lindstrom J, Ohlsson A: Intra-osseous anchorage of dental prostheses. I. Experimental studies. Scand J Plast Reconstr Surg 3: 81-100, 1969

Candel-Marti E, Carrillo-Garcia C, Penarrocha-Oltra D, Penarrocha-Diago M: Rehabilitation of atrophic posterior maxilla with zygomatic implants: review. J Oral Implantol 38: 653-657, 2012

Canny J: A computational approach to edge detection. IEEE Trans Pattern Anal Mach Intell 8: 679-698, 1986

Chappard D, Retailleau-Gaborit N, Legrand E, Basle MF, Audran M: Comparison insight bone measurements by histomorphometry and microCT. J Bone Miner Res 20: 1177-1184, 2005

Chrcanovic BR, Pedrosa AR, Neto Custodio AL: Zygomatic implants: a critical review of the surgical techniques. Oral Maxillofac Surg 17: 1-9, 2013
Corbella S, Taschieri S, Del Fabbro M: Long-Term outcomes for the treatment of atrophic posterior maxilla: a systematic review of literature. Clin Implant Dent Relat Res, 2013

Corvello PC, Montagner A, Batista FC, Smidt R, Shinkai RS: Length of the drilling holes of zygomatic implants inserted with the standard technique or a revised method: a comparative study in dry skulls. J Craniomaxillofac Surg 39: 119-123, 2011

Davo R, Malevez C, Pons O: Immediately loaded zygomatic implants: a 5-year prospective study. Eur J Oral Implantol 6: 39-47, 2013

Del Fabbro M, Rosano G, Taschieri S: Implant survival rates after maxillary sinus augmentation. Eur J Oral Sci 116: 497-506, 2008

Doube M, Klosowski MM, Arganda-Carreras I, Cordelieres FP, Dougherty RP, Jackson JS, et al: BoneJ: free and extensible bone image analysis in imageJ. Bone 47: 1076-1079, 2010

Esposito M, Worthington HV: Interventions for replacing missing teeth: dental implants in zygomatic bone for the rehabilitation of the severely deficient edentulous maxilla. Cochrane Database Syst Rev, 2013 9: CD004151

Fanuscu MI, Chang TL: Three-dimensional morphometric analysis of human cadaver bone: microstructural data from maxilla and mandible. Clin Oral Implant. Res 15: 213-218, 2004

Ferrigno N, Laureti M, Fanali S, Grippaudo G: A long-term follow-up study of nonsubmerged ITI implants in the treatment of totally edentulous jaws. Part I: tenyear life table analysis of a prospective multicenter study with 1286 implants. Clin Oral Implant. Res 13: 260-273, 2002

Goiato MC, Pellizzer EP, Moreno A, Gennari-Filho H, dos Santos DM, Santiago JF] et al: Implants in the zygomatic bone for maxillary prosthetic rehabilitation: a systematic review. Int J Oral Maxillofac Surg 43: 748-757, 2014

Hildebrand T, Ruegsegger P: Quantification of bone microarchitecture with the structure model Index. Comput Methods Biomech Biomed Engin 1: 15-23, 1997

Kato Y, Kizu Y, Tonogi M, Ide Y, Yamane G: Observation of the internal structure of the zygomatic bone by micro-computed tomography. J Oral Biosci 46: 523-529, 2004

Kato Y, Kizu Y, Tonogi M, Ide Y, Yamane GY: Internal structure of zygomatic bone related to zygomatic fixture. J Oral Maxillofac Surg 63: 1325-1329, 2005

Kim JE, Shin JM, Oh SO, Yi WJ, Heo MS, Lee SS, et al: The three-dimensiona microstructure of trabecular bone: analysis of site-specific variation in the human jaw bone. Imaging Sci Dent 43: 227-233, 2013a

Kim S, Macdonald HM, Nettlefold L, McKay HA: A comparison of bone quality at the distal radius between Asian and white adolescents and young adults: an HRpQCT study. J Bone Miner Res 28: 2035-2042, 2013b

Liu XS, Walker MD, McMahon DJ, Udesky J, Liu G, Bilezikian JP, et al: Better skeletal microstructure confers greater mechanical advantages in Chinese-American women versus white women. J Bone Miner Res 26: 1783-1792, 2011 
Malevez C, Abarca M, Durdu F, Daelemans P: Clinical outcome of 103 consecutive zygomatic implants: a 6-48 months follow-up study. Clin Oral Implant. Res 15: $18-22,2004$

Migliorança R, Ilg JP, Serrano AS, Souza RP, Zamperlini MS: Sinus exteriorization of the zygoma fixtures: a new surgical protocol. Implant News 3: 30-35, 2006

Moon HS, Won YY, Kim KD, Ruprecht A, Kim HJ, Kook HK, et al: The threedimensional microstructure of the trabecular bone in the mandible. Surg Radiol Anat 26: 466-473, 2004

Nkenke E, Hahn M, Lell M, Wiltfang J, Schultze-Mosgau S, Stech B, et al: Anatomic site evaluation of the zygomatic bone for dental implant placement. Clin Ora Implant. Res 14: 72-79, 2003

Pjetursson BE, Tan K, Lang NP, Bragger U, Egger M, Zwahlen M: A systematic review of the survival and complication rates of fixed partial dentures (FPDs) after an observation period of at least 5 years. Clin Oral Implant. Res 15: 667-676, 2004

Putman MS, Yu EW, Lee H, Neer RM, Schindler E, Taylor AP, et al: Differences in skeletal microarchitecture and strength in African-American and white women. J Bone Miner Res 28: 2177-2185, 2013

Romeed SA, Malik R, Dunne SM: Zygomatic implants: the impact of zygoma bone support on biomechanics. J Oral Implantol 40: 231-237, 2014

Schneider CA, Rasband WS, Eliceiri KW: NIH image to Imagej: 25 years of image analysis. Nat Methods 9: 671-675, 2012

Stella JP, Warner MR: Sinus slot technique for simplification and improved orientation of zygomaticus dental implants: a technical note. Int J Oral Maxillofac Implant. 15: 889-893, 2000
Uchida Y, Goto M, Katsuki T, Akiyoshi T: Measurement of the maxilla and zygoma as an aid in installing zygomatic implants. J Oral Maxillofac Surg 59: 1193-1198, 2001

Ulm C, Kneissel M, Schedle A, Solar P, Matejka M, Schneider B, et al: Characteristic features of trabecular bone in edentulous maxillae. Clin Oral Implant. Res 10: 459-467, 1999

Ulm C, Tepper G, Blahout R, Rausch-Fan X, Hienz S, Matejka M: Characteristic features of trabecular bone in edentulous mandibles. Clin Oral Implant. Res 20: 594-600, 2009

Ulm CW, Kneissel M, Hahn M, Solar P, Matejka M, Donath K: Characteristics of the cancellous bone of edentulous mandibles. Clin Oral Implant. Res 8: 125-130, 1997

Van Steenberghe D, Malevez C, Van Cleynenbreugel J, Bou Serhal C, Dhoore E, Schutyser F, et al: Accuracy of drilling guides for transfer from threedimensional CT-based planning to placement of zygoma implants in human cadavers. Clin Oral Implant. Res 14: 131-136, 2003

Weischer T, Schettler D, Mohr C: Titanium implants in the zygoma as retaining elements after hemimaxillectomy. Int J Oral Maxillofac Implant. 12: 211-214, 1997

Weng D, Jacobson Z, Tarnow D, Hurzeler MB, Faehn O, Sanavi F, et al: A prospective multicenter clinical trial of $3 i$ machined-surface implants: results after 6 years of follow-up. Int J Oral Maxillofac Implant. 18: 417-423, 2003

Yoshino M, Kato Y, Kizu Y, Tonogi M, Abe S, Ide Y, et al: Study on internal structure of zygomatic bone using micro-finite element analysis model-differences between dentulous and edentulous dentition in Japanese cadavers. Bull Tokyo Dent Coll 48: 129-134, 2007 